\title{
Fake news e infodemia científica durante la Covid-19, ¿dos caras de la misma crisis informacional?
} Fake news and the scientific infodemic during
Covid-19: two faces of the same informational crisis? Alexandre López-Borrull

López-Borrull, Alexandre (2020). "Fake news e infodemia científica durante la Covid-19, ¿dos caras de la misma crisis informacional?". Anuario ThinkEPI, v. 14, e14e07.

https://doi.org/10.3145/thinkepi.2020.e14e07

Publicado en IweTe/ el 14 de diciembre de 2020

\begin{abstract}
Alexandre López-Borrull
https://orcid.org/0000-0003-1609-2088

Universitat Oberta de Catalunya

Estudis de Ciències de la Informació i de la Comunicació

alopezbo@uoc.edu
\end{abstract}

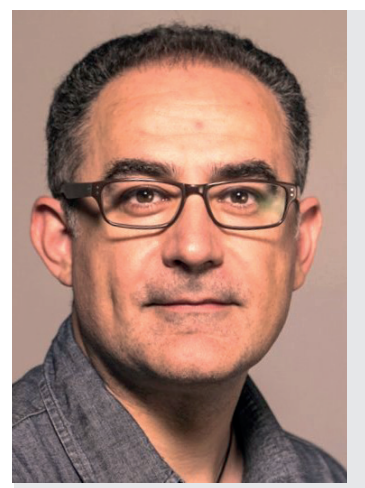

Resumen: El presente artículo parte de la actual crisis sanitaria de la Covid-19 para reflexionar sobre los efectos a nivel de uso de información, principalmente por la existencia de las fake news y también los problemas de la comunicación científica que en cierto sentido la crisis ha agravado. Así como la propia OMS habla de una infodemia, en este caso hablamos también de una infodemia científica. Ello nos sitúa en la reflexión sobre si ha producido también una crisis informacional, de confianza y de seguridad. Se propone una mayor alfabetización científica dada la mayor presencia de contenidos científicos en el debate y opinión social.

Palabras clave: Fake news; Desinformación; Crisis informacional; Negacionismo científico; Infodemia científica; Covid-19.

Abstract: Considering the current health crisis related to Covid-19, this article reflects on its effects at the level of information use, mainly due to the existence of fake news as well as the problems of scientific communication which it has aggravated. Indeed, like the infodemic mentioned by the WHO itself, herein we consider a scientific infodemic. This leads to a debate regarding whether this has also produced an informational, trust, and security crisis. Greater scientific literacy is proposed given the increased presence of scientific content in the social debate and opinion.

Keywords: Fake news; Disinformation; Informational crisis; Scientific denial; Scientific infodemic; Covid-19.

\section{Introducción}

Esta nota ThinkEPI se ha escrito a finales de 2020, a medio camino entre la segunda ola y una nueva incertidumbre. Sí, no me atrevo a llamarlo de otra forma. Más que nunca y en relación con la Covid-19, la fecha de escritura de nuestros artículos debe considerarse. Sin duda, la palabra de este año debería ser pues, incertidumbre, necesaria en cualquier digestión para reflexionar sobre lo que está ocurriendo, lo que ha ocurrido, e incluso para intentar prever lo que pueda ocurrir. Todo ello con la intención no solo de prevenir nuevas crisis, sino para tener al final un relato común y compartido que podamos evaluar, entender y mejorar. 
Según mi punto de vista, existen múltiples aspectos para describir lo que ha sucedido durante la pandemia y que tendrían relación con una visión informacional de la sociedad. De hecho, son suficientemente relevantes como para que los profesionales de la información y la comunicación los podamos asumir como algo nuestro, algo propio, y nos podamos posicionar, e incluso lo podamos convertir en nuestro objeto de estudio.

Posiblemente muchos hemos echado en falta una mayor presencia social y mediática de nuestro colectivo en momentos en que la buena información era clave para la toma acertada de decisiones. Al fin y al cabo, se trata de nuestra materia prima, la información, la que está siendo creada, manipulada (en todos los sentidos), atacada e incluso "conspiracionalizada", si ello existe, aunque ustedes ya me entenderán. Seguramente en muchos ámbitos podríamos haber encontrado un camino para poder dar nuestra opinión. Por ejemplo, podemos preguntarnos si hemos sabido mejorar y mitigar los flujos de desinformación en las redes sociales.

Asimismo, también cabe preguntarnos si la información ha pasado de ser únicamente un activo para poder ser un freno para el desarrollo a considerar ante cualquier ola (o tsunami) de desinformación. $\mathrm{O}$, incluso, si quizá desde nuestra disciplina estamos sabiendo liderar el debate sobre nuestro papel en la verificación y el control en la gestión y digestión de los medios sociales. Finalmente, en algo que también atañe desde hace tiempo a nuestra disciplina, valorar si la sociedad está suficientemente preparada para las nuevas formas de difusión de la información y el conocimiento científico que se están generando y consolidando, algunas de las cuales en línea con la ciencia abierta y otras más propias del marketing académico.

En este sentido, hay que destacar que partimos de la premisa de que nos encontramos también ante una crisis informacional. De la gran seguridad de que nos encontrábamos creando y consumiendo contenidos, hemos pasado a un escenario donde no teníamos la información que necesitábamos para tomar decisiones acertadas, porque no existía y la que nos llegaba podría ser contradictoria y preliminar. Deberemos, pues, estudiar si esta sensación de inseguridad con relación a la información, esta crisis informacional, se va a unir a la crisis sanitaria y económica. Sobre todos estos temas, intentaremos, pues, lanzar unas cuantas ideas, pero también generar dudas y plantear preguntas.

\section{Las fake news, como las mascarillas, forman ya parte del día a día de la Covid-19}

En el lejano 2019 ya alertamos de cómo la existencia de las fake news y su traslación a la información científica podrían ser un problema refiriéndonos a ello como fake science, en relación con el aumento de contenidos falsos de tipo científico (López-Borrull; Ollé, 2019). En marzo de 2020 una vez iniciada la crisis por la covid-19 es importante señalar que ya la propia Organización Mundial de la Salud (OMS), además de la lógica pandemia, nos alertaba de lo que llamó infodemia, la existencia de una gran cantidad de información, difícil de digerir, mucha de la cual además era falsa (López-Borrull; Ollé, 2020). Tal como la crisis se ha planteado, me refiero a la infodemia científica, porque mucha de esta desinformación que campa por todos los medios sociales y nefastos medios de comunicación se refiere a contenidos de tipo científico, tanto sobre el origen de la Covid-19 como aspectos de cura, prevención o próximamente las vacunas.

Por mi parte, debo decir que en los primeros días de confinamiento no esperaba gastar tanto tiempo leyendo tantos inventos, fantochadas, magufadas, cuñadismos y conspiranoias diversas como hemos podido recibir, leer e incluso desmentir. Uso estos nuevos modismos por no llamarlos directamente mentiras, porque también es cierto que en todo relato negacionista aparecen unos puntos intermedios que son ciertos, para vestir mejor la desinformación. Tener un profesional de la información de cabecera debería haber sido un lujo en esta era de complejidad informacional.

Cabe decir, en este sentido, que los intentos de eliminar las fake news y la desinformación en los medios de comunicación han estado más presentes que nunca y que ya la desinformación era apuntada por Europa como uno de los problemas y amenazas para la propia democracia (Comisión Europea, 2018). Pero sin duda la crisis provocada por la Covid-19 ha llevado a un incremento exponencial de noticias falsas que ha generado un cambio en nuestras formas de consumo de información, como ya se está describiendo de forma acertada en la bibliografía (Masip et al., 2020; Moscadelli et al., 2020; Salaverría et al., 2020). Sin duda, va a ser uno de los campos de estudio más interesantes en los próximos años y ya se está viendo en la gran cantidad de monográficos, artículos y libros que empiezan a

“No esperábamos gastar nuestro escaso tiempo leyendo tantos inventos, fantochadas, magufadas, cuñadismos y conspiranoias diversas como publicarse sobre el tema. 
Aun así, por mucho que los estudiosos y académicos estemos empezando a entender y hacer propuestas para corregir el estado de desinformación continuo en el que a veces nos hemos movido, cabe tener en cuenta también cómo han ido evolucionando las propias fake news. No estamos hablando de un debate complejo como sería la necesidad del uso de mascarillas en el espacio público, algo en lo que los científicos están aportando evidencias a marchas forzadas y por tanto podría ser debatible. Hablamos sobre todo de negacionismo científico de gran calidad, como reflejan por ejemplo productos audiovisuales como Plandemic, Un crimen oculto o Hold-up.

https://www.genbeta.com/redes-sociales-y-comunidades/plandemic-video-conspiranoico-coronavirusque-facebook-youtube-otras-plataformas-tratan-eliminar

https://maldita.es/malditaciencia/2020/10/02/documental-un-crimen-oculto/

https://elpais.com/television/2020-11-20/el-conspiracionismo-sobre-el-virus-desembarca-en-francia-conel-documental-hold-up.html

Hablamos de contenidos de buena factura, bien presentados, y que ofrecen una plausibilidad elevada y una gran difusión por la sencillez en sus respuestas, y por dar certezas allí donde el discurso comunicativo oficial parece dubitativo cuando no contradictorio (Elías, 2020).

Producciones repletas de datos, algunos falsos y otros verdaderos pero descontextualizados, se presentan difíciles de desmontar para un ciudadano estándar, y ello hace surgir la duda sobre si estamos suficientemente preparados para combatir el negacionismo científico. Sin duda, como profesionales de la información podrían ser competencias un tanto oxidadas. Sin embargo,

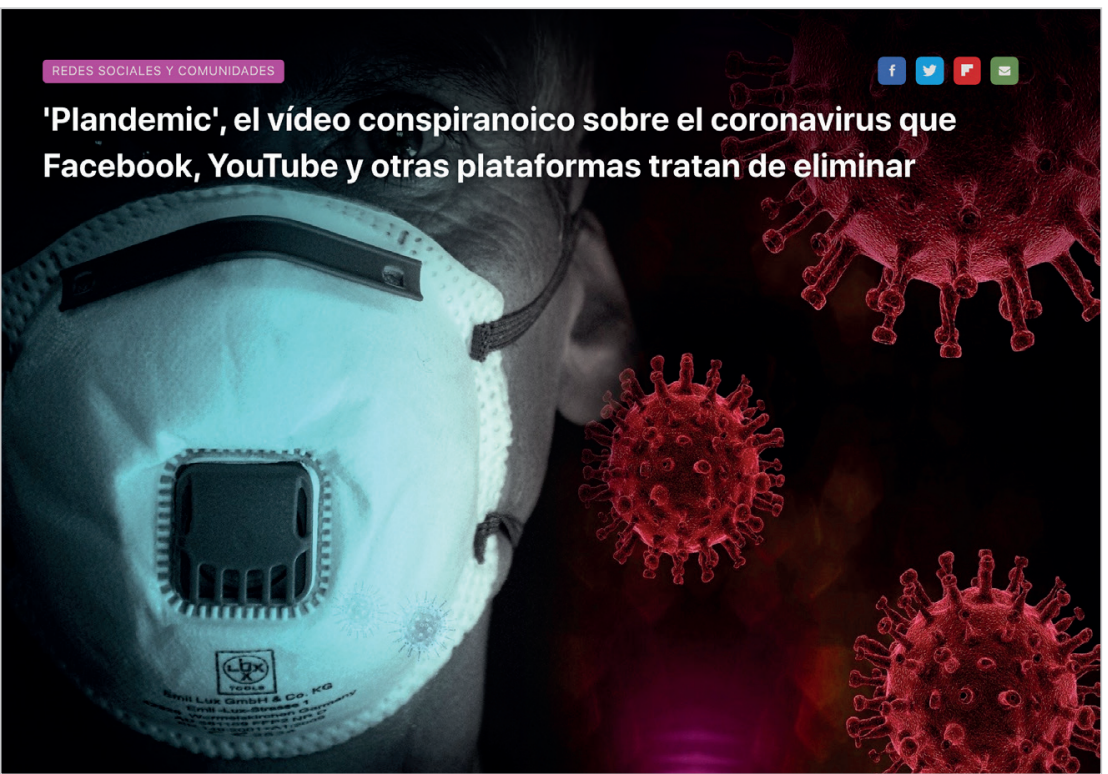

https://www.genbeta.com/redes-sociales-y-comunidades/plandemic-video-conspiranoico-coronavirus-que-facebook-youtube-otras-plataformas-tratan-eliminar

esta crisis ha hecho que debamos replantearlas, sobre todo para no dar por hecho que en la sociedad de la información y el conocimiento existe una mayoría de personas que saben encontrar la información pertinente y filtrar la impertinente. Esto ya no es lo que era al principio de siglo, donde la clave estaba en saber utilizar los motores de búsqueda, sino que ahora es necesario saber interpretar el ecosistema informativo de medios y redes sociales. Así, el problema ya no es solo el ruido sino la desinformación. Sin duda, un nuevo reto en el que los profesionales de la información debemos reafirmarnos más que nunca, aunque por la especialización de la temática debamos hacerlo en colaboración con científicos, divulgadores y curadores de contenidos (López-Borrull; Vives-Gràcia; Badell, 2018).

Finalmente, por poner un ejemplo de la complejidad del momento, recordar cómo una nota de Horton (2020), editor jefe de The lancet, dio gasolina a diversos bulos al afirmar que según su punto de vista la Covid-19 no era una pandemia. Sí, este era el título del artículo que parecía cuestionar la crisis sanitaria. Evidentemente, cuando se leía el artículo, Horton venía a decir que de hecho más que pandemia deberíamos hablar de "sindemia", en el sentido de que coexisten distintas epidemias o brotes de enfermedades y todas ellas causan la actual crisis sanitaria. Pero ya había dado pie a múltiples olas de desinformación porque una revista científica parecía negar la pandemia. Posiblemente, deberemos ser mucho más cautos a la hora de redactar. Solo nos faltaría caer en el clickbait académico.

\section{Ejemplos de infodemia científica: viejos problemas, nuevas incertidumbres}

Anteriormente hemos visto cómo con la pandemia de la Covid-19 ha circulado una cantidad ingente de desinformación científica, de forma intencionada o no. En este apartado veremos casos que ejemplifican, a mi entender, algunos de los problemas de la comunicación científica y que se han visto acuciados en esta crisis informacional.

De la misma forma que los expertos dicen que la educación virtual no es lo mismo que la educación que se ha llevado a cabo durante el periodo de confinamiento, también deberemos evaluar si lo que 
se ha llevado a cabo en abierto no es necesariamente ciencia abierta, aunque se le parezca más que nunca. Podríamos hablar de una competitividad en abierto más que de una colaboración significativa. La lucha contra la Covid-19 no ha sido un proceso como el del proyecto Genoma Humano sino una nueva carrera espacial, geopolítica y con trazos de guerra fría, como afirmaba Priego en agosto (2020). Ello puede ser corroborado por la gran cantidad de posibles vacunas que van a aparecer y a la velocidad pandémica que se habrá llevado a cabo, en los términos usados por Lurie et al. (2020).

Más que nunca, las administraciones, los profesionales sanitarios y la sociedad han pedido (de hecho, exigido) respuestas rápidas para preguntas nuevas, preguntas que aún no habían sido formuladas. En esta carrera por aportar evidencias, los países, los financiadores y las revistas han sabido entender las prioridades y se han llevado a cabo toda una serie de acuerdos y declaraciones en la línea de poner la información en abierto tan pronto como fuera posible.

https://es.unesco.org/covid19/communicationinformationresponse/opensolutions

Por supuesto, las revistas depredadoras han seguido haciendo negocio (Shelomi, 2020).

Como era previsible, la crisis pandémica ha centrado y ocupado muchas de las investigaciones de los últimos meses, y autores como Teixera da Silva, Tsigaris y Erfanmanesh (2020) cifraban que los seis primeros meses se habían publicado casi 24.000 artículos en revistas presentes en la Web of Science y en Scopus. Tiempo habrá, y será necesario, para describir la calidad o las posibles redundancias que se hayan producido por solapamiento de metodologías y estudios (Elgendy et al., 2020).

\section{1. "Revísame despacio, que tengo prisa"}

Teniendo en cuenta la crisis sanitaria, es obvio que científicos, autores y editores debían hacer un esfuerzo para poder compartir lo más rápido posible conocimiento científico. Con la prontitud, podía pasar que la revisión por pares no fuera el auténtico proceso guardián de la ciencia en los términos descritos por Crane (1967). Así, con la cantidad de artículos publicados puede pensarse casi en términos de error experimental, pero como veremos en el siguiente ejemplo, un error en la aceptación y publicación de un artículo puede ser relevante, y lo fue. Cabe destacar, como siempre, el papel de grupos como Retraction Watch y su papel en compilar ya más de 40 artículo retirados total o temporalmente.

https://retractionwatch.com/retracted-coronavirus-covid-19-papers/

Por su parte, Soltani y Patini (2020) han llevado a cabo un pequeño estudio preliminar de los artículos retirados en relación con la Covid-19 como un efecto colateral del esfuerzo de investigación realizado.

El caso más famoso de artículos retirados y que han tenido mayor impacto mediático y global, han sido los dos publicados por The lancet y The new England journal of medicine sobre el uso de medicamentos como la hidroxicloroquina como tratamiento eficaz contra la Covid-19 y con Sapan Desai como uno de los autores. Recordemos que estas dos revistas publicaron sendos artículos donde Surgisphere, la compañía de Desai, proporcionaba toda una serie de datos sobre el uso del tratamiento en centenares de hospitales que validaban sus conclusiones. Una vez publicados, se elevaron quejas inmediatas acerca de la validez de los datos de investigación que llevaban a dichas conclusiones, por la incapacidad real de poder haber tenido acceso a tanta cantidad de información y protocolos. Se inició una investigación requiriendo los datos a la empresa. Ante la imposibilidad de acceder a los datos primarios que validasen las conclusiones, se retiraron los artículos (Piller; Servick, 2020).

https://www.thelancet.com/pdfs/journals/lancet/PIIS01406736(20)31180-6.pdf

En el caso de uno de los medicamentos, la hidroxicloroquina, el impacto mediático fue aún mayor por cuanto incluso el presidente Trump había hablado de él en sus comparecencias públicas. La propia OMS inicialmente paró las investigaciones sobre el medicamento

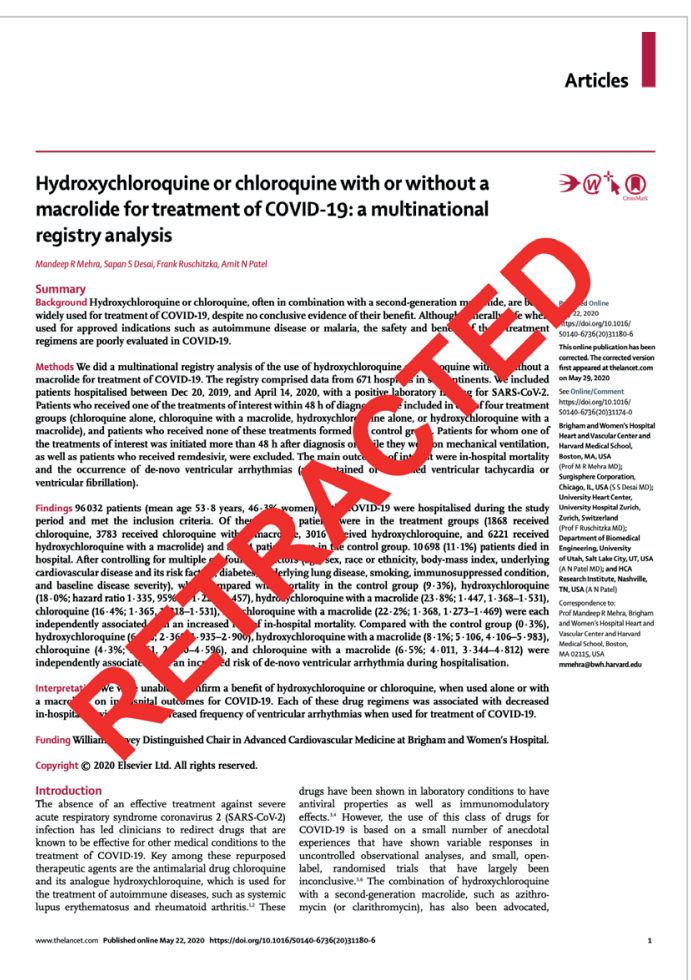

https://www.thelancet.com/pdfs/journals/lancet/ PIIS0140-6736(20)31180-6.pdf 
después de la publicación de los artículos. Tiempo después, se ha ido demostrando que no aporta un valor específico contra la enfermedad por mucho que Trump y Bolsonaro lo defendieran como viable. https://www.eldiario.es/catalunya/oriol-mitja-hidroxicloroquina-beneficios-covid-19_1_6021617.html

Aunque a nivel comunicativo se trató de un error importante y que puede ser usado por negacionistas para defender que las revistas científicas no tienen la verdad absoluta (como afirman), científicos como O'Brian (2020) justamente defienden que la ciencia tiene sus procesos de verificación, y que, si no funcionan en el proceso de publicación, pueden funcionar en el de post-publicación, y, por tanto, debe tenerse más confianza en el sistema de comunicación científica.

\section{2. ¿Debemos citar la piel del oso antes de que sea publicada?}

También me parece significativo, en la línea de intentar explicar los flujos de información científica entre el mundo académico y el resto de la sociedad, el artículo que fue publicado el 13 de junio en medRxiv y que aún no ha sido publicado en ninguna revista científica (Chavarria-Miró et al., 2020). Por tanto, se trata de un preprint tal y como el propio repositorio describe. Desde mi punto de vista, este ha sido uno de los principales puntos ciegos del intercambio de información científica y su divulgación en medios tradicionales.

El manuscrito recoge, de forma sorprendente para todos, incluso quizá para los propios autores, que en aguas residuales recogidas en la ciudad de Barcelona ya había presencia del SARS-CoV-2 en 2019. Sin ser experto en el tema no voy a profundizar en el tema en cuestión considerando la reputación de los autores, lo importante creo que es entender algunos aspectos de los flujos comunicativos y de la importancia de sus resultados. En primer lugar, el artículo estuvo dos semanas en el repositorio sin ninguna repercusión mediática, hasta que el día 26 de junio se hizo un comunicado de prensa por parte de la universidad. A las pocas horas ya era noticia a nivel internacional.

https://www.ub.edu/web/ub/ca/menu_eines/noticies/2020/06/042.html

Cabe entender que las repercusiones de la validez de dicho artículo son muy elevadas, incluso a nivel geopolítico. Así, se ha usado y citado como respuesta a los prejuicios y la xenofobia del "virus chino" tal y como el presidente Trump no deja de llamar al SARS-CoV-2 (Zeng; Wang; Zhang, 2020). Así, un preprint alcanza una viralización y unas métricas de visualización y presencia en las redes sociales muy elevadas. Evidentemente, es importante que los resultados y las conclusiones sean ciertas, pero a nivel comunicativo lo es más que

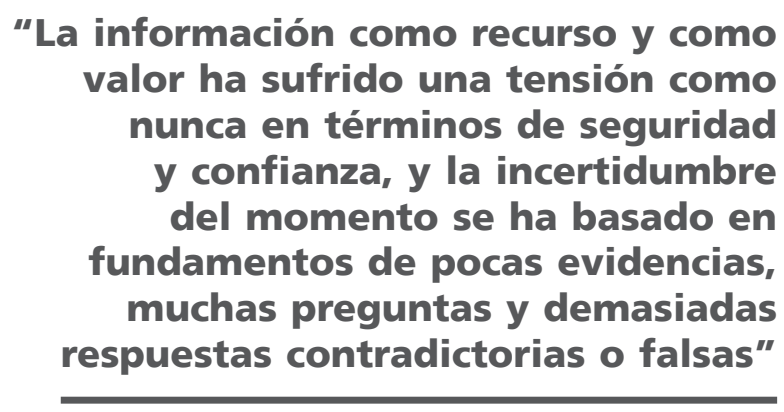
se exprese adecuadamente en qué estado está el artículo. Salomone desde SINC (2020) ha realizado una interesante radiografía al artículo y los problemas que contiene desde un punto de vista científico, sobre todo porque parece apuntar al problema de la reproducibilidad al no quedar muestra de las aguas residuales congeladas.

\subsection{Cuando la geopolítica también publica artículos}

Los movimientos negacionistas y los conspiracionistas no son siempre compartimentos cerrados, sino que aprenden del entorno. Sin duda, han aprendido que una de las estrategias habituales en la negación del cambio climático, las vacunas o el creacionismo pasa por la amplificación de cualquier artículo científico publicado o prepublicado que ponga en duda el discurso mayoritario de los investigadores.

El ejemplo que presentamos se refiere al conocido como The Yan Report, por la viróloga china Li-Meng Yan, que ha sido depositado en Zenodo (Yan et al., 20201). En él, se mantiene que el SARSCoV-2 es una modificación y creación artificial. Yan se fugó de China en abril y siempre ha manifestado que el país asiático ha censurado y encubierto buena parte de lo sucedido en el inicio de la pandemia. https://nypost.com/2020/07/10/chinese-virologist-flees-after-accusing-beijing-of-covid-19-cover-up/

Medios como la Fox han amplificado su discurso y ha servido al gobierno Trump para justificar sus dudas y críticas a lo que el presidente sigue llamando el "virus chino". De este episodio, más allá de que la mayor parte de científicos y verificadores le dan poco crédito, lo significativo desde mi punto de vista son dos aspectos.

https://maldita.es/malditaciencia/2020/11/01/estudio-virologa-china-li-meng-yan-coronavirus-creadolaboratorio-covid-pandemial

En primer lugar, que la fuente de financiación y la institución declarada por los autores es la Rule 
of Law Society, una organización con sede en Nueva York presidida por Steve Bannon, que fue señalado como uno de los principales estrategas de las elecciones ganadas por Donald Trump en 2016, y gran especialista en la creación de fake news a través de su anterior portal Breitbart News.

En segundo lugar, para fijarnos en las métricas, hay que resaltar que dicho informe en el repositorio ha sido visto más de 950.000 y ha sido descargado más de 700.000 ocasiones. Además, cuando se busca en Google Scholar, aunque ha sido relativamente poco citado, dispone de 46 versiones distintas en múltiples y desconocidos servidores. Sin duda, son aspectos donde se entremezclan la comunicación científica y la viralización de fake news, por lo que debemos considerar que no siempre serán vasos impermeables.

\section{4. ¿Qué papel podemos jugar los profesionales de la información ante una crisis informacional? Propuesta por una mayor alfabetización científica}

Hemos querido usar algunos ejemplos ilustrativos para explicar de forma preliminar que de la pandemia deberemos analizar y entender muchos de los aspectos que han tenido lugar. Según mi punto de vista, podemos hablar también de que se ha producido una crisis informacional con relación a que la información como recurso y como valor ha sufrido una tensión como nunca en términos de seguridad y confianza, y que la incertidumbre del momento también se ha basado en fundamentos de pocas evidencias, muchas preguntas y demasiadas respuestas contradictorias o falsas.

Así, una pandemia con redes sociales es otro tipo de vivencia y, además de aquellas cosas buenas que haya tenido, podemos también reflejar aspectos negativos, sobre todo en lo referente a la calidad de la información. Si la crisis económica de 2008 potenció la información económica y ello nos condujo a una mayor alfabetización en economía y conceptos como la prima de riesgo y otros formaron parte de forma constante del ecosistema informativo, en esta ocasión el ecosistema se ha llenado de conocimientos científicos como la Rt o la incidencia a 14 días, por poner algún ejemplo. Asimismo, científicos como Fernando Simón, Oriol Mitjà o Antoni Trilla han pasado a ser sujetos mediáticos (López-Borrull; Ollé, 2020).

Por otra parte, la existencia de una crisis informacional nos debería llevar a una reflexión e incluso un debate sobre cómo una mayor alfabetización científica puede ayudar al progreso, en un momento en que la ciencia está tomando un papel activo y un protagonismo social y político relevante, como por ejemplo el posicionamiento inédito de ciertas revistas científicas en las elecciones americanas (Csiszar, 2020). No hablamos solo de entender las fuentes, sino de aprender a saber qué es un preprint, cómo divulgar o cómo curar contenidos, y entender que la cultura científica es un elemento de desarrollo básico para capear los retos del futuro. En este sentido, luchar contra el negacionismo y la mentira debe ser equilibrado por los profesionales de la información con las libertades de expresión y la transparencia informativa, de la misma forma que llevamos haciendo con relación a la propiedad intelectual y el acceso a la información.
"Luchar contra el negacionismo y la mentira debe ser equilibrado por los profesionales de la información con las libertades de expresión y la transparencia informativa, de la misma forma que llevamos tiempo haciendo con la propiedad intelectual y el acceso a la información" 
Finalmente, recordar el papel que los profesionales de la información pueden llevar a cabo en relación con la desinformación, porque de la misma forma que con la gestión de datos masivos, estamos capacitados no solo para entender y describir la veracidad de algo, sino también para analizar el contexto, el ecosistema informativo y las consecuencias éticas y morales de su uso y abuso. Es sin duda un camino abierto en los próximos años.

\section{Notas}

1. Aunque ha habido dudas, se ha preferido seguir citando la fuente, aunque no se confíe en la veracidad de su contenido

\section{Referencias}

Chavarria-Miró, Gemma; Anfruns-Estrada, Eduard; Guix, Susana; Paraira, Miquel; Galofré, Belén; Sánchez, Gloria; Pintó, Rosa; Bosch, Albert (2020). "Sentinel surveillance of SARS-CoV-2 in wastewater anticipates the occurrence of COVID-19 cases". MedRxiv, 13 June.

https://doi.org/10.1101/2020.06.13.20129627

Comisión Europea (2018). La lucha contra la desinformación en línea: un enfoque europeo. Comunicación de la Comisión al Parlamento Europeo, al Consejo, al Comité Económico y Social Europeo y al Comité de las Regiones. https://eur-lex.europa.eu/legal-content/ES/TXT/PDF/?uri=CELEX:52018DC0236\&from=en

Crane, Diana (1967). "The gatekeepers of science: Some factors affecting the selection of articles for scientific journals". American sociologist, v. 32, p. 195-201.

https://www.jstor.org/stable/27701277

Csiszar, Alex (2020). "Scientific journals are denouncing Trump. That's Normal". Wired, 20 October. https://www.wired.com/story/scientific-journals-are-denouncing-trump-thats-normal

Elgendy, Akram Y.; Barakat, Amr F.; Ibrahim, Joseph; Alkukhun, Laith; Mamas, A.; Mamas, B. M.; Elgendy, Islam Y. (2020). "The landscape of medical literature in the era of Covid-19: Original research versus opinion pieces". Journal of general internal medicine v. 35, p. 2813-2815.

https://doi.org/10.1007/s11606-020-06021-8

Elías, Carlos (2020). “Expertos/as científico/as y comunicación gubernamental en la era de las fake news. Análisis de la estrategia informativa del Covid-19 en España". Prisma social, n. 31, pp. 7-39.

https://revistaprismasocial.es/article/view/3945/4537

Horton, Richard (2020). "Offline: Covid-19 is not a pandemic". The lancet, v. 396, n. 10255, P874.

https://doi.org/10.1016/S0140-6736(20)32000-6

López-Borrull, Alexandre; Ollé, Candela (2019). "La curación de contenidos científicos como respuesta a las noticias y a la ciencia falsas". Anuario ThinkEPI, v. 13, e13e07.

https://doi.org/10.3145/thinkepi.2019.e13e07

López-Borrull, Alexandre; Ollé, Candela (2020). “Curación de contenidos científicos en tiempos de fake science y Covid-19: una aproximación entre las ciencias de la información y la comunicación". En: Comunicación y diversidad. Selección de comunicaciones del VII Congreso Internacional de la Asociación Española de Investigación de la Comunicación (AE-IC). Valencia, España, 28-30 de octubre, pp. 281-289. EPI SL. ISBN: 9788412023954 https://doi.org/10.3145/AE-IC-epi.2020.e16

López-Borrull, Alexandre; Vives-Gràcia, Josep; Badell, Joan-Isidre (2018). "Fake news, ¿amenaza u oportunidad para los profesionales de la información y la documentación?". El profesional de la información, v. 27, n. 6, pp. 1346-1356.

https://doi.org/10.3145/epi.2018.nov.17

Lurie, Nicole; Saville, Melanie; Hatchett, Ricard; Halton, Jane (2020). "Developing Covid-19 vaccines at pandemic speed". The new England journal of medicine, v. 382, n. 21.

https://www.nejm.org/doi/full/10.1056/NEJMp2005630

Masip, Pere; Aran-Ramspott, Sue; Ruiz-Caballero, Carlos; Suau, Jaume; Almenar, Ester; Puertas-Graell, David (2020). "Consumo informativo y cobertura mediática durante el confinamiento por el Covid-19: sobreinformación, sesgo ideológico y sensacionalismo". El profesional de la información, v. 29, n. 3, e290312.

https://doi.org/10.3145/epi.2020.may.12

Moscadelli, Andrea; Albora, Giuseppe; Biamonte, Massimiliano-Alberto; Giorgetti, Duccio; Innocenzio, Michele; Paoli, Sonia; Lorini, Chiara; Bonanni, Paolo; Bonaccorsi, Guglielmo (2020). "Fake News and Covid-19 in Italy: Results of a quantitative observational study". International journal of environmental research and public health, v. 17, n. 16, 5850.

https://doi.org/10.3390/ijerph17165850 
O'Brian, Mark R. (2020). "Retractions and controversies over coronavirus research show that the process of science is working as it should". The conversation, July 6.

https://theconversation.com/retractions-and-controversies-over-coronavirus-research-show-that-the-process-of-science-is-working-as-it-should-140326

Piller, Charles; Servick, Kelly (2020). "Two elite medical journals retract coronavirus papers over data integrity questions". Science, June 4.

https://www.sciencemag.org/news/2020/06/two-elite-medical-journals-retract-coronavirus-papers-over-data-integrity-questions

Priego, Alberto (2020). "Geopolítica de las vacunas: la nueva 'carrera espacial'". Cinco días, 28 agosto. https://cincodias.elpais.com/cincodias/2020/08/27/opinion/1598523779_185515.htm/

Salaverría, Ramón; Buslón, Nataly; López-Pan, Fernando; León, Bienvenido; López-Goñi, Ignacio; Erviti, María-Carmen (2020). "Desinformación en tiempos de pandemia: tipología de los bulos sobre la Covid-19". El profesional de la información, v. 29, n. 3, e290315.

https://doi.org/10.3145/epi.2020.may.15

Salomone, Mónica G. (2020) "Qué sabemos sobre la presencia del coronavirus en aguas de Barcelona recogidas en marzo de 2019". SINC, 26 junio.

https://www.agenciasinc.es/Reportajes/Que-sabemos-sobre-la-presencia-del-coronavirus-en-aguas-de-Barcelona-recogidas-en-marzo-de-2019

Shelomi, Matan (2020). "Opinion: Using Pokémon to detect scientific misinformation". The scientist, November 1. https://www. the-scientist.com/critic-at-large/opinion-using-pokmon-to-detect-scientific-misinformation-68098?utm_content=145325857\&utm_medium=social\&utm_source=twitter\&hss_channel=tw-18198832\&s=09

Soltani, Parisa; Patini, Romeo (2020). "Retracted Covid-19 articles: A side-effect of the hot race to publication". Scientometrics, v. 125, p. 819-822.

https://doi.org/10.1007/s11192-020-03661-9

Teixeira-da-Silva, Jaime A.; Tsigaris, Panagiotis; Erfanmanesh, Mohammadamin (2020). "Publishing volumes in major databases related to Covid-19". Scientometrics, first online.

https://doi.org/10.1007/s11192-020-03675-3

Yan, Li-Meng; Kang, Shu; Guan, Jie; Hu, Shanchang (2020). “Unusual features of the SARS-CoV-2 genome suggesting sophisticated laboratory modification rather than natural evolution and delineation of its probable synthetic route". Zenodo.

http://doi.org/10.5281/zenodo.4028830

Zeng, Guangting; Wang, Linlin; Zhang, Zanling (2020). "Prejudice and xenophobia in Covid-19 research manuscripts". Nature human behaviour, v. 4, 879.

https://doi.org/10.1038/s41562-020-00948-y

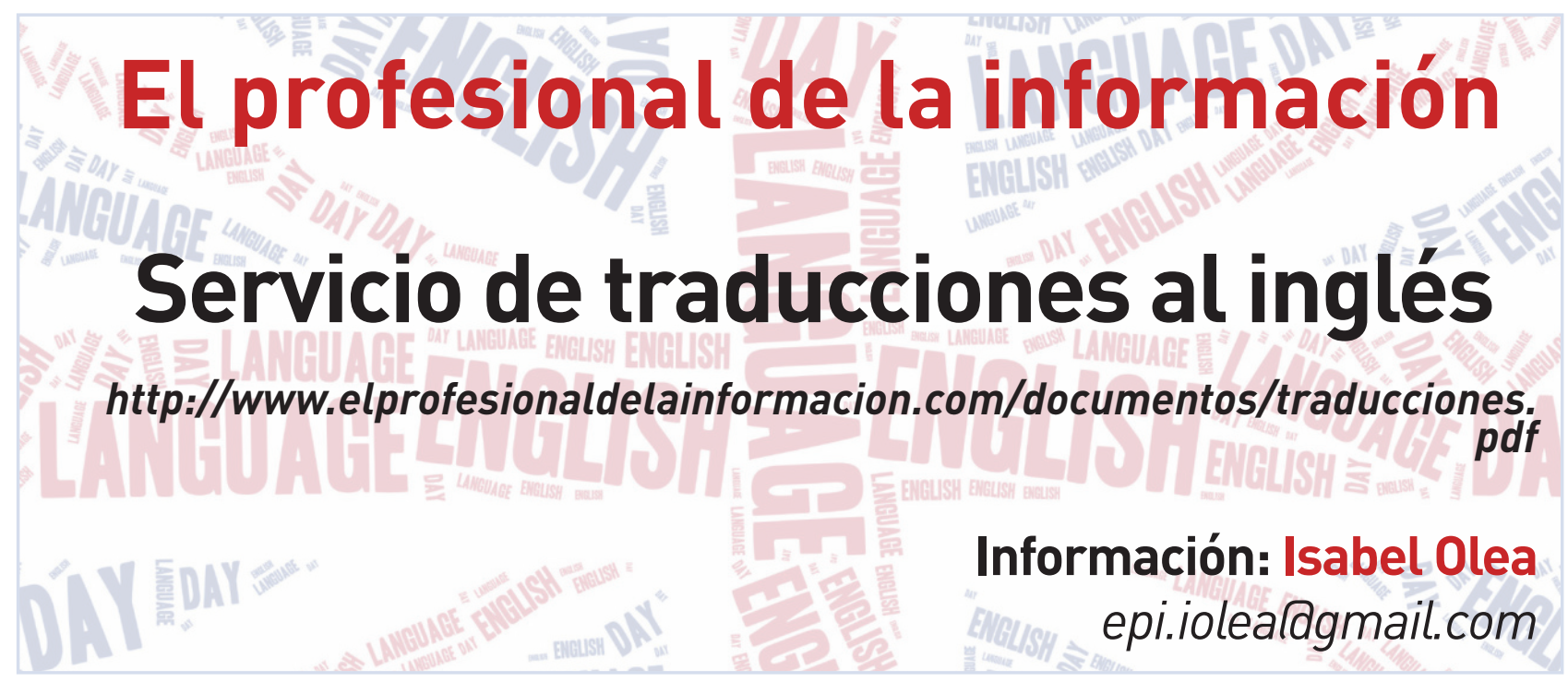

